\title{
Oncologic Safety and Clinical Outcomes of Skin and Nipple Sparing Mastectomy with Immediate Definite Implant Reconstruction in Breast Cancer Patients using Superolateral Radial Incision: A Prospective Study
}

\author{
Ahmed Gamal El-Din Osman, MD, MRCS; Mohamed Said Gali, MD, MRCS \\ Department of General Surgery, Ain Shams University, Egypt
}

The expansion of total skin-sparing mastectomy (SSM) criteria over time has allowed greater numbers of patients to experience the aesthetic and psychological benefits that SSM and nipple-sparing mastectomy (NSM) approach provided. However, we have to weigh the oncologic safety, and the postoperative complication. Superolateral radial incision provides an exploratory incision with minimal complication.

Patients and methods: This study was a prospective study conducted on 28 female patients with invasive breast cancer requiring mastectomy who underwent skin-sparing mastectomy and nipple sparing mastectomy via the superolateral radial incision.

Conclusion: Skin-sparing mastectomy and nipple-sparing mastectomy via superolateral radial incision is an oncologically safe procedure with a low-postoperative complication rate and an excellent esthetic outcome.

Key words: Skin-sparing mastectomy, nipple sparing mastectomy, implant.

\section{Introduction}

According to The National Cancer Institute data, the breast is the most common site of cancer in Egyptian women. Breast cancer accounts for $38.8 \%$ of total malignancies among Egyptian females. Moreover, it is an important cause of death among Egyptian women. ${ }^{1}$

Skin-sparing mastectomy (SSM) aims at removing the breast tissue while saving the breast skin. A natural skin envelope is thus created, that is filled with a breast implant or with the patient's own tissue from another part of the body. Skin-sparing mastectomies significantly improve the cosmetic outcome of surgery and give the best option for breast reconstruction and recovery. If the nipple has not been invaded by cancer and can be saved as well, the surgeon would then be able also to perform a nipple-sparing mastectomy (NSM). ${ }^{2}$

The choice of incision is important in SSM and NSM to avoid nipple ischemia and necrosis. The blood supply of the nipple is derived medially from the internal mammary perforators, superiorly from the thoracoacromial artery, the vessels to the serratus anterior, and the lateral thoracic artery, laterally from the terminal branches of the $3^{\text {rd }} 8^{\text {th }}$ intercostal perforators, and the inferiorly from branches of the superior epigastric artery. Approximately $60 \%$ of the total breast blood supply is derived from the robust perforators of the internal mammary artery, which may explain the increased rate of nipple necrosis when using a medial incision. Unnecessary damage to the peripheral blood supply of the breast envelope may also occur if the dissection is carried too far beneath the inframammary fold, lateral to the latissimus dorsi muscle, or over the sternum; therefore, these areas should be avoided. ${ }^{3}$

Our initial experience with skin-sparing mastectomy (SSM) utilized different incisions such as inframammary incision, radial, and axillary incisions. We now prefer the superolateral radial incision to avoid possible damage of a significant source of blood supply to the nipple, and consequently avoiding nipple ischemia. Our aim was to focus on skin-sparing mastectomy and immediate reconstruction with definite implant via a superolateral radial incision as an ideal incision for mastectomy and to determine the oncological safety and cosmetic outcome of this procedure.

\section{Patients and methods}

The current study was conducted on 28 female patients with invasive breast cancer requiring mastectomy admitted to Ain Shams University Hospitals in the period between March 2013 and January 2016. The age of the patients ranged between 34 and 49 years. Exclusion criteria included the presence of distant metastases, a history of previously treated ipsilateral breast cancer, inflammatory tumors, and smoking at the time of surgery. Patients initially presenting with skin involvement who had a good response to neoadjuvant chemotherapy and no longer had tumor involvement of the skin at the time of 
mastectomy were not excluded from the study. Because nipple-sparing mastectomy (NSM) could be difficult and might lack cosmetic appeal in patients with significantly ptotic breasts. Breast size and ptosis were taken into consideration in the decision to offer NSM.

Demographic data were collected from all patients. All patients were offered routine blood tests, mammography, breast ultrasound, FNAC, and metastatic workup before operations. Tumor sizes were assessed by preoperative mammogram and sonogram.

The excised masses were labeled and sent to frozen pathology assessment for the adequacy of the resection margins. Patients with persistent positive superficial margin were subjected to excision of the skin above the tumor. In patients who were offered NSM, the tissue underneath the NAC was sent for frozen pathological assessment to determine the involvement of NAC with cancer. If the result was positive, the patient was shifted to SSM, the weight of the surgical specimen was also reported.

All patients signed a written consent to undergo oncoplastic techniques and the possible need for contralateral reduction mastopexy to get a better aesthetic result was explained to all patients. Patients' medical records were reviewed in multidisciplinary meetings to determine the therapeutic strategy.

\section{Surgical technique}

\section{Outlining the breast mound}

Minimizing the length of the incision created a challenge in identifying anatomical landmarks within the breast envelope during the dissection. The boundaries of the breast mound were outlined with a marking pen prior to incision (Figure 1) to assist in identifying the boundaries at the periphery of the breast tissue during the dissection.

\section{The incision}

A superolateral radial incision (Figure 2) was used through which mastectomy and axillary clearance were done. In patients who required removal of the NAC and overlying skin, an extension of the incision to encircle the areola was preferred allowing excellent exposure. We typically avoided the medial incision so as not to disrupt the abundant blood supply to the nipple from the internal mammary perforators as was demonstrated in Crowe's experience. ${ }^{4}$ Moreover, reconstruction with implants is extremely difficult through a medial incision. (Figure 2).
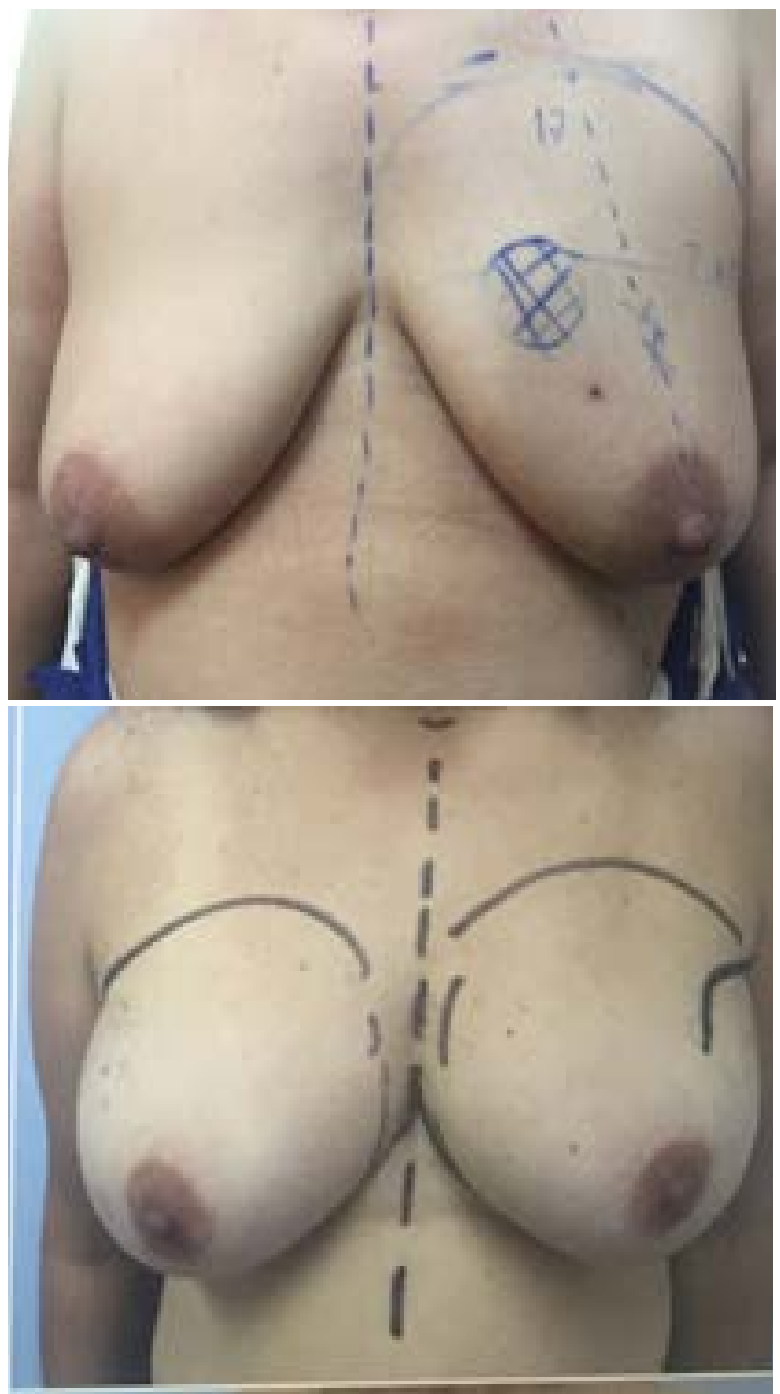

Fig 1: Marking of boundaries of the breast mound prior to incision while the patient in erect position.

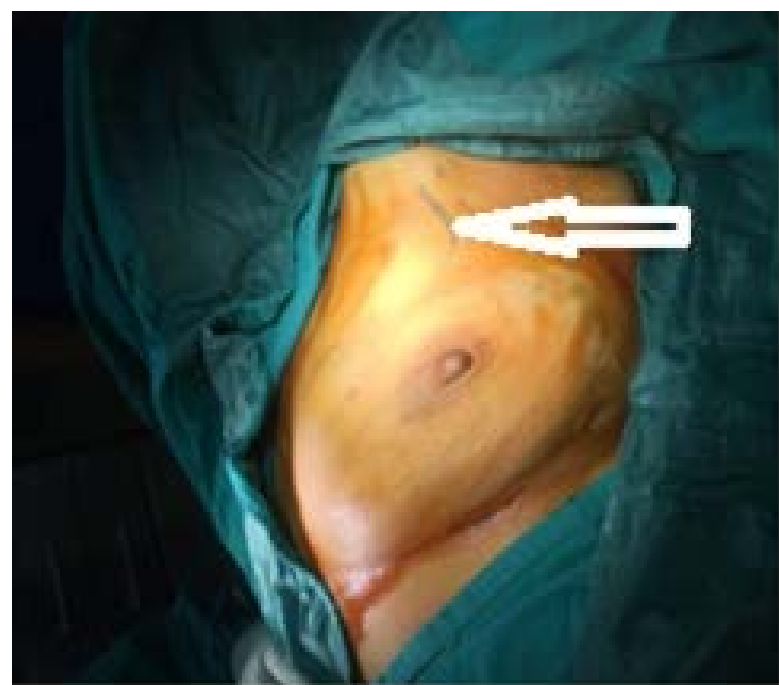

Fig 2: Lateral incision directing toward areola.

We deepened the incision into the superficial plane of subcutaneous tissue, the margins of the skin flap were held upward using skin hooks with the 
surgeon applying counter pressure using the left index and middle fingers in the same manner as for ordinary mastectomy. The breast was dissected from the pectoralis major muscle, starting on the medial side and progressing laterally to the margins of the pectoralis major muscle. After the margins of the pectoralis major muscle were freed, the whole breast was inverted out from wound and removed in the same manner as during ordinary mastectomy (Figure 3). The breast tissue under the NAC was examined histologically on intraoperative frozen pathology.

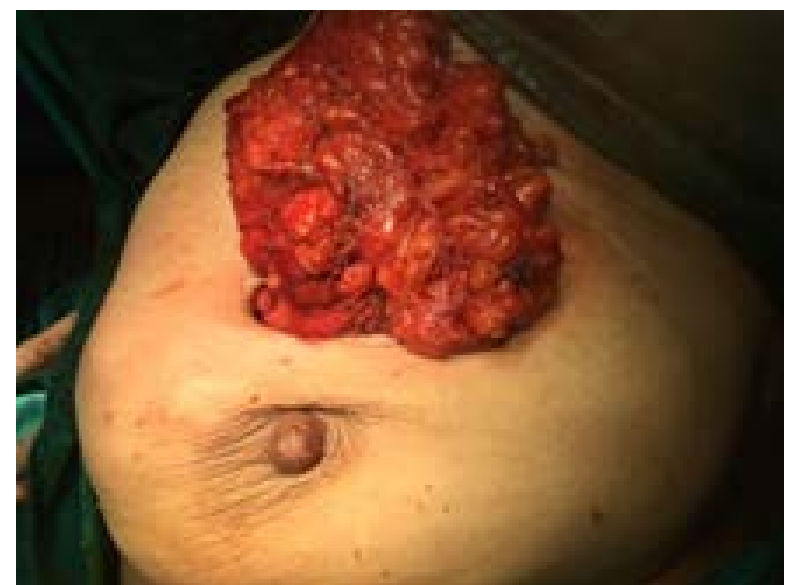

Fig 3: The whole breast tissue is excised and delivered through the incision.

If it was involved, NAC was also excised, thereby converting from NSM to SSM. Complete axillary lymph node dissection (ALND) was carried out from the same incision. A subpectoral pocket was gently created under direct vision with a lighted retractor using a long electrocautery while lifting up the pectoralis major muscle using an arm retractor. The pocket extended superiorly to the second rib and medially to the sternum, and the lower extent of the pocket was created to match the contralateral inframammary fold. The implant was inserted into the subpectoral pocket (Figure 4A). Finally, the bleeding points were carefully coagulated by electrocautery. A suction drain was inserted into the axilla and breast.

Follow-up visits took place after 7 (Figure 4b) and 15 days, and at 1, 2, 3, 4, and 6 months after surgery, then once a year for 5 years. The primary endpoint of the study was the development of tumor recurrence within 15 months after surgery. The secondary endpoint was the viability of skin within 6 months after surgery. Necrosis was defined as the loss of breast skin vitality requiring nursing care for secondary wound healing or surgery for debridement or cutaneous graft, Skin necrosis was defined to be minimal when the surface size of necrosis was less than $2 \mathrm{~cm}^{2}$, moderate when it was $2-10 \mathrm{~cm}^{2}$, or large when it was more than 10 $\mathrm{cm}^{2}$.
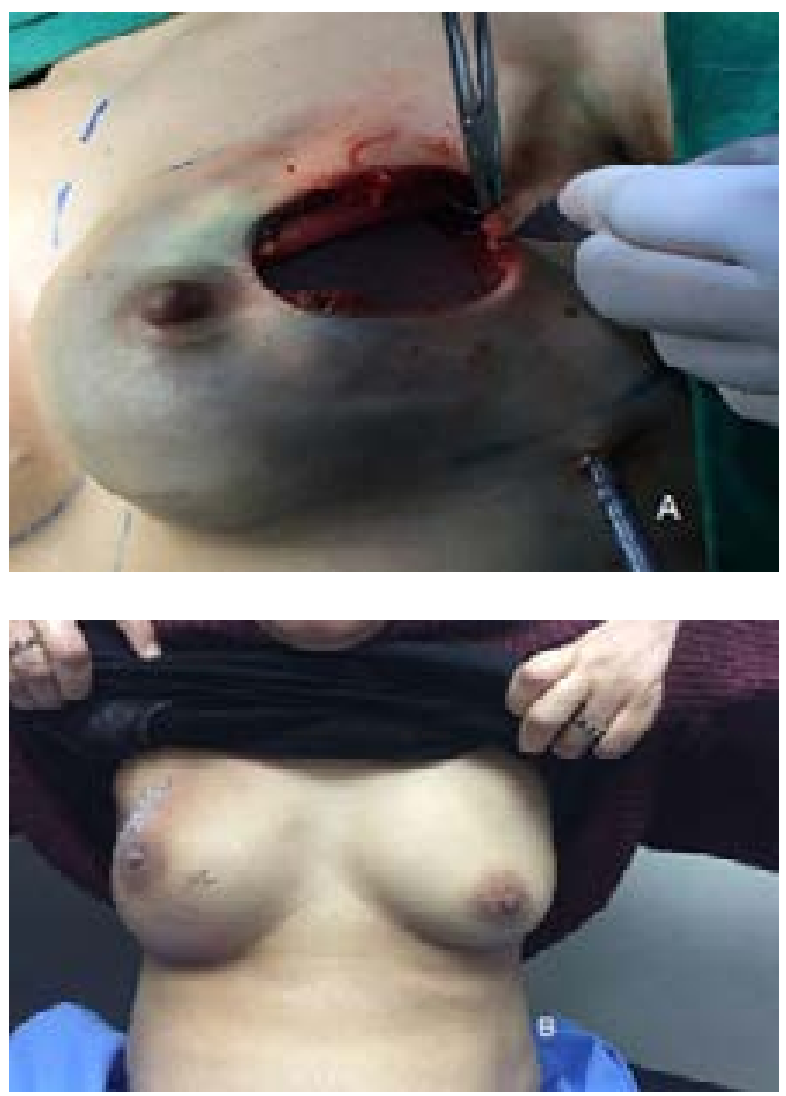

Fig 4A: Implant inserted in subpectoral pocket, 4B: 7 days post operative.

At follow-up, patients were also assessed for other local complications such as hematoma, infections, and capsular contraction by clinical examination, laboratory assays, and imaging, as appropriate. Also, the evolution of necrosis was assessed depending on whether or not it was cured at 6 months, and the duration of dressing and any revision surgery was described.

\section{Statistics}

Variables were described using mean, standard deviation, median and range for quantitative parameters, and count and percentages for categorical parameters. In order to identify factors associated with a high risk of necrosis, we analyzed, in univariate analysis, the mammary volume (estimated by bra size), and comorbidities [body mass index (BMI) and smoking history]. Variables associated with a significant $p$-value in univariate analysis were selected for entering into a multivariate analysis model, using a backward elimination method with a significance value set up at the 0.05 level.

\section{Results}

The mean age of the patient was $47 y$, the mean BMI was 28. One patient was a smoker and nine patients (32\%) had co-morbidities in the form of diabetes mellitus and hypertension that were controlled at the time of surgery. Nineteen patients $(67.8 \%)$ initially had NSM, four of them 
were converted to SSM because of suspected NAC involvement on frozen sections. Postoperatively, the removed NAC was found to be histologically involved in $3(75 \%)$ of 4 patients, nine patients (32.1\%) underwent SSM. The main patient characteristics are presented in Table 1. Two patients underwent mastectomy for diffuse in situ disease and 26 patients had an invasive carcinoma, 9 of them were locally advanced T3 and T4. All final surgical margins and retroareolar tissue samples were clear of residual disease. Reconstruction was performed using prostheses in all patients, with a mean prosthesis volume of $273 \pm 84 \mathrm{~mm}^{3}$. All patients underwent axillary clearance except $2(7.1 \%)$ patients with diffuse DCIS diagnosed preoperatively with true cut biopsy and confirmed intraoperatively with frozen histopathology.

Postoperatively, axillary lymph nodes were histologically positive in 20 cases $(71 \%)$ and negative in 6 cases (29\%). Postoperatively, all patients underwent standard adjuvant therapy based on risk category and biology of the tumor. A total of 4 patients $(16 \%)$ received radiotherapy (RT) as part of adjuvant therapy. 21 patients received hormonal therapy $(75 \%)$. The median follow-up duration was 30 months.

One patient $(3.5 \%)$ developed local recurrence which was diagnosed as invasive duct carcinoma by true cut biopsy and she underwent excision of the skin envelope with the removal of the implant (modified radical mastectomy). Three patients $(10.5 \%)$ had postoperative skin necrosis; two cases of less than $2 \mathrm{~cm}^{2}$, one case between 2 and $10 \mathrm{~cm} .^{2}$ Those patients required dressings for one and half months. Two cases of skin necrosis were located in the periareolar area, and the medium-size skin necrosis occurred around the incision. Two cases suffered from mild infection around the wound which healed by frequent dressing within 15 days postoperative and only one case suffered from hematoma which resolved spontaneously without any surgical interference. Capsular contraction occurred in 9 (32\%) patients, 4 of them were grade I and II which required no intervention, 3 of them were grade III which were treated with lipo-filling and 2 of them were grade IV. Those 2 particular patients received radiotherapy postoperative $p=0.0005$ and they were treated surgically by capsulotomy and lipofilling. The following risk factors were studied: smoking, breast size, and BMI, none of which were associated with skin necrosis in univariate analysis (Table 2).
Table 1: Baseline characteristics of patients $\mathbf{n}=\mathbf{2 8}$

\begin{tabular}{|c|c|}
\hline \multicolumn{2}{|l|}{ Age at diagnosis } \\
\hline Mean \pm SD & $47 \pm 7.4$ \\
\hline Median & $46(34-49)$ \\
\hline \multicolumn{2}{|l|}{ BMI, kg/m2 } \\
\hline Mean $\pm S D$ & $28 \pm 3.7$ \\
\hline Median & $27(19-43)$ \\
\hline $\mathrm{BMI} \leq 20$ & 1 \\
\hline $\mathrm{BMI} \geq 30$ & 6 \\
\hline \multicolumn{2}{|l|}{ Bra Size } \\
\hline Small & 4 \\
\hline Moderate & 8 \\
\hline Large & 16 \\
\hline Past smoker & 3 \\
\hline \multicolumn{2}{|l|}{ Tumor size in mm } \\
\hline Mean \pm SD & $32 \pm 17.3$ \\
\hline Median & $28(8-57)$ \\
\hline \multicolumn{2}{|l|}{ Clinical tumor stage } \\
\hline T0 & 0 \\
\hline Tis & 2 \\
\hline $\mathrm{T} 1$ & 1 \\
\hline $\mathrm{T} 2$ & 15 \\
\hline T3 & 9 \\
\hline T4 & 1 \\
\hline No & 2 \\
\hline N1 & 16 \\
\hline \multicolumn{2}{|l|}{ Histological type } \\
\hline Invasive duct carcinoma & 12 \\
\hline $\begin{array}{l}\text { Invasive lobular carci- } \\
\text { noma }\end{array}$ & 10 \\
\hline IDC and ILC & 4 \\
\hline DCIS & 2 \\
\hline \multicolumn{2}{|l|}{$\begin{array}{l}\text { Hormonal status of } \\
\text { tumor }\end{array}$} \\
\hline ER-/PR- & 7 \\
\hline $\mathrm{ER}-/ \mathrm{PR}+$ & 1 \\
\hline $\mathrm{ER}+/ \mathrm{PR}-$ & 5 \\
\hline $\mathrm{ER}+/ \mathrm{PR}+$ & 15 \\
\hline \multicolumn{2}{|l|}{ HER2 status } \\
\hline HER2+ & 7 \\
\hline HER 2- & 21 \\
\hline \multicolumn{2}{|l|}{ Luminal classification } \\
\hline Luminal A & 4 \\
\hline Luminal B & 17 \\
\hline Basal type & 5 \\
\hline HER 2 type & 2 \\
\hline
\end{tabular}


Table 2: Factors associated with necrosis in univariate analysis

\begin{tabular}{lcc}
\hline \multicolumn{1}{c}{ Necrosis } & P-value \\
\hline History of smoking & & \\
Yes & 1 & 0.1 \\
No & 4 & \\
Bra size & & \\
Small & 0 & \\
Moderate & 2 & 0.2 \\
Large & 3 & \\
BMI & & \\
$\leq 30$ & 1 & 0.1 \\
$\geq 30$ & 4 & \\
\hline
\end{tabular}

Table 3: Post-operative complication

\begin{tabular}{ll}
\hline Complication & \\
\hline Early & 1 \\
Haematoma & 2 \\
Infection & \\
Late & 3 \\
Skin necrosis & 9 \\
Capsular contraction & 1 \\
Grade I & 3 \\
Grade II & 3 \\
Grade III & 2 \\
Grade IV & 1 \\
Recurrence &
\end{tabular}

\section{Discussion}

The expansion of total skin-sparing mastectomy criteria over time has allowed greater numbers of patients to experience the aesthetic and psychological benefits that SSM and NSM approach provided. However, we have to weigh the oncologic safety as our primary endpoint designed, particularly for patients presenting with extensive disease. Our patient population included a significant number of patients with locally advanced disease $n=10(35.7 \%)$, nearly all of whom were offered NSM approaches unless they had tumor involvement of the nipple who were offered SSM. Review of our outcomes for these patients demonstrates low rates of local recurrence only one patient $3.5 \%$ which was comparable with Munhoz et al, ${ }_{1}^{4}$ who evaluated 106 patients with breast cancer treated with SSM with a mean followup of 65.5 months. They found local recurrences (LR) in $3.7 \%$ of patients and non involved of the spared NAC. Our result is nearly similar to Gonzalez and Rancati ${ }^{5}$ who compared SSM and MRM which did not reveal a statistically significant difference in local recurrence rates. With a mean of 68 months, local recurrences were observed in $5.4 \%$ in the
SSM group versus $5.1 \%$ in the group of MRM. When Gonzalez and Rancati analyzed the LR rate of patients who underwent SSM and compare it with the rates reported in randomized prospective studies of mastectomies without reconstruction, relapses were found to occur in $2-10 \%$ of patients with a follow-up of 6-10 years; these figures are comparable to those of reconstructive procedures that retain skin. Our result with all these studies supports the oncological safety of this procedure.

Our secondary endpoint was the rate of skin necrosis and capsular contraction, complication rates are difficult to compare between studies because this parameter depends on multiple factors, such as reconstruction techniques, surgeon's experience, and previous and subsequent treatments. In our study 3 patient suffered from skin necrosis ( $11 \%$ of patients), 2 of them were mild and periareolar, both patients were from NSM group and healed within 20 days while one case was moderate and required one and half month of dressing and follow up till complete healing. However, despite the high incidence of chemotherapy and radiotherapy, we reported only a single case with moderate skin necrosis 3.7\% which was treated conservatively. Munhoz et $\mathrm{al}^{6}$ reported worse results than ours using either hemi-periareolar or double concentric periareolar incisions with the incidence of local wound dehiscence and partial flap necrosis of $(12.8 \%$ and $10.3 \%$ ) and (4.5\% and $4.5 \%)$, respectively. Regolo et $\mathrm{al}^{7}$ reported an NAC necrotic complication rate of $59.7 \%$ in 32 NSMs. Our $100 \%$ survival of NAC and implant may be due to our cautious standardized maneuver and a good selection of cases as we avoided current smokers and uncontrolled comorbidities as well as using safe superolateral radial incision with no interference with medial blood supply. As for capsular contraction, the Royal Marsden series ${ }^{8}$ found a clear association of capsular contracture with RT $(p<0.001)$ but did not find any evidence of association with other variables including chemotherapy and tamoxifenin Cox univariate regression. The Bristol series ${ }^{11}$ also found a clear association of capsular contraction (CC) with radiotherapy (RT) $(p=0.048)$, but no evidence of a chemotherapy or hormone therapy effect. This study shows a statistically and clinically significant higher rate of severe capsular contraction in patients who received postoperative RT compared to those who did not. Our study showed 9 patients $32 \%$ with capsular contraction, 2 of them were grade III who were treated by lipofilling and the other 2 were severe (grade IV) that required surgical intervention. All of these patients received post operative RT. Burdge ${ }^{10}$ studied a large series of patients with significant rates of adjuvant chemotherapy and radiation therapy have shown necrotic complication rates in the range of 
$5-20 \%$, which is similar to rates seen in this series. Certainly, postmastectomy radiation therapy is a major contributor to reconstructive complications particularly in the setting of implant reconstruction. Prior studies of patients undergoing SSM and postmastectomy radiation therapy have shown complication rates as high as $30 \% .{ }^{11,12}$ Despite the increased risk of complications, our protocol involves offering immediate reconstruction routinely to all patients, regardless of radiation status, given the acceptable rates of complications and high rates of successful reconstruction seen even for patients who receive postmastectomy radiation therapy. ${ }^{13}$ However, for patients with a high likelihood of needing postmastectomy radiation therapy, we try to achieve the option of breast conservation whenever possible through approaches such as the use of neoadjuvant chemotherapy to minimize complications. ${ }^{14}$ Neoadjuvant chemotherapy can help to reduce the need for postmastectomy radiation therapy because many patients with a significant response to treatment, even those initially presenting with advanced disease, can achieve good oncologic outcomes without radiation therapy, ${ }_{1}^{15,16}$ or avoid mastectomy altogether and achieve breast conservation. ${ }^{17}$ These results will be of value in counseling women about options for reconstruction, and may influence women who will require postoperative RT to opt for totally autologous reconstructions. Where possible another novel technique of delayed immediate breast reconstruction utilizes an expander only at the time of the skin-sparing mastectomy (to preserve the skin envelope) followed by further surgery 6-9 months later using latissmus dorsi (LD) flap and permanent implant. This has the advantage of using the unirradiated tissue but exposes the patient to poorer initial cosmoses and a more extensive operation when recovering from the long cancer journey. Our 3 patients $10.7 \%$ with necrosis rate is comparable to Carlson et al. ${ }^{18}$ 118 SSMs were performed with various types of immediate reconstruction, a retrospective Danish study evaluated the complications of SSM associated with implants in 141 patients (208 breasts). ${ }^{19}$ The global complication rate was $20 \%$, with infection, epidermolysis, and necrosis occurring in 10,10 , and $13 \%$ of cases, respectively. Most patients were operated for prophylactic reasons. Skin necrosis was significantly more frequent in cancer patients $(21.3 \%)$ than in prophylactic mastectomy $(7.5 \%)$, and more frequent in the smoking group (3/38 compared with $17 / 164 ; p=0.05$ ). In the study by Downes et al, ${ }^{20} 92 \%$ of patients received chemotherapy postoperatively and $65 \%$ had adjuvant RT. Only two patients (5.3\%) had skin necrosis. Transverse rectus abdominis myocutaneous (TRAM) flap, LD flap, and implants were used for reconstruction in 81.6, 7.9, and $10.5 \%$ of cases, respectively. Our study could not identify risk factors associated with necrosis due to the very low number of events; however, several risk factors such as smoking, breast size, obesity, and previous chest wall irradiation were identified in other studies. ${ }^{21}$ On the other hand, post-mastectomy irradiation is associated with a high rate of surgical complications. When postmastectomy RT is indicated, major complications appear less frequent with autologous tissue than with implants, as shown by Berry et al., who concluded that musculocutaneous flap is the best option for patients who received neoadjuvant $\mathrm{RT}^{22}$

Thus, we acknowledge that this low rate of necrosis has been obtained in selected patients and selected sites and might not be extrapolated to an unselected population.

\section{Conclusion}

National Comprehensive Cancer Network $(\mathrm{NCCN}-2016)^{23}$ outlined that, SSM is a safe procedure that provides good cosmetic results with good local cancer control. Multidisciplinary evaluation with an experienced surgical team with proper patient selection are essentials for the success of the operation. In appropriately selected patients, SSM and nipple-sparing mastectomy via superolateral radial incision are an oncologically safe procedure with a low-postoperative complication rate and an excellent esthetic outcome. Nevertheless, longer term follow-up is needed to evaluate capsular contracture. The benefits of single-stage reconstruction, such as lower costs and reduced hospitalization time for the patient, make the use of this procedure attractive option for selected patients.

\section{Reference}

1. Ibrahim $A$, Khaled $H$, Mikhail $N$, Kamel $H$, Baraka $\mathrm{H}$ : Cancer incidence in Egypt: Results of the National Population-Based Cancer Registry Program. Journal of Cancer Epidemiology 2014; Article ID 437971, 215-232.

2. González, Eduardo G, Alberto O: Skin-sparing mastectomy. Gland Surgery 2015; 4: 541-553.

3. Stolier AJ, Sullivan SK, Dellacroce FJ: Technical considerations in nipple-sparing mastectomy: 82 consecutive cases without necrosis. Ann Surg Oncol 2008; 15: 1341-7.

4. Munhoz $\mathrm{AM}$, Aldrighi $\mathrm{CM}$, Montag $\mathrm{E}$, Arruda EG, Aldrighi JM, Gemperli R, et al: Clinical outcomes following nipple-areola sparing mastectomy with immediate implant-based breast reconstruction: A 12-year experience with an analysis of patient and breast-related 
factors for complications. Breast Cancer Res Treat 2013; 140: 545-555.

5. González EG, Rancati AO: Skin-sparing mastectomy. Gland Surg 2015; 4: 541-553.

6. Munhoz AM, Aldrighi C, Montag E: Optimizing the nipple areola sparing mastectomy with double concentric periareolar incision and bidimensional expander-implant reconstruction: Aesthetic and technical refinements. Breast 2009; 18: 356-367.

7. Regolo L, Ballardini B, Gallarotti E: Nipple sparing mastectomy: An innovative skin incision for an alternative approach. Breast 2008; 17: 8-11.

8. Behran wala KA, Dua RS, Ross GM, Ward A, $A^{\prime}$ Hern R, Gui GP: The influence of radiotherapy on capsule formation and aesthetic outcome after immediate breast reconstruction using biodimensional anatomical expander implants. J Plast Reconstr Aesthet Surg 2006; 59: 10431051.

9. Thomson $\mathrm{HJ}$, Potter $\mathrm{S}$, Greenwood RJ, Bahl A, Barker J, Cawthorn SJ, et al: A prospective longitudinal study of cosmetic outcome in immediate latissimus dorsi breast reconstruction and the influence of radiotherapy. Ann Surg Oncol 2008; 15: 10811091.

10. Burdge EC, Yuen J, Hardee M: Nipple skinsparing mastectomy is feasible for advanced disease. Ann Surg Oncol 2013; 20: 3294-3302.

11. Spear SL, Shuck J, Hannan L, Albino F, Patel $\mathrm{K}$ : Evaluating long-term outcomes following nipple-sparing mastectomy and reconstruction in the irradiated breast. Plast Reconstr Surg 2014; 133: 605e-614e.

12. Warren Peled A, Foster RD, Ligh C, Esserman LJ, Fowble B, Sbitany H: Impact of total skin-sparing mastectomy incision type on reconstructive complications following radiation therapy. Plast Reconstr Surg 2014; 134: 169-175.

13. Warren Peled A, Foster RD, Esserman LJ, Hwang ES, Fowble B: Increasing the time to expander-implant exchange after postmastectomy radiation therapy reduces expander-implant failure. Plast Recon Surg 2012; 130: 503-509.
14. Warren A, Sbitany H, Foster RD, Esserman LJ: Oncoplastic mammoplasty as a strategy for reducing reconstructive complications associated with postmastectomy radiation therapy. Breast 2014; 20: 302307.

15. Warren A, Wang F, Stover AC: Selective use of postmastectomy radiation therapy in the neoadjuvant setting. San Antonio Breast Cancer Symposium, San Antonio, TX Dec 2012.

16. Fowble BL, Einck JP, Kim DN: Role of postmastectomy radiation after neoadjuvant chemotherapy in stage II-III breast cancer. Int J Radiat Oncol Biol Phys 2012; 83: 494-503.

17. Cho JH, Park JM, Park HS: Oncologic safety of breast conserving surgery compared to mastectomy in patients receiving neoadjuvant chemotherapy for locally advanced breast cancer. J Surg Oncol 2013; 108: 531-536.

18. Carlson GW, Losken A, Moore B: Results of immediate breast reconstruction after skinsparing mastectomy. Ann Plast Surg 2001; 46: 222-228.

19. Petersen A, Eftekhari AL, Damsgaard TE: Immediate breast reconstruction: A retrospective study with emphasis on complications and risk factors. J Plast Surg Hand Surg 2012; 46: 344- 348.

20. Downes KJ, Glatt BS, Kanchwala SK: Skin-sparing mastectomy and immediate reconstruction is an acceptable treatment option for patients with high-risk breast carcinoma. Cancer 2005; 103: 906-913.

21. Delbaere M, Delaporte T, Toussoun G, Delay E: Skin-sparing mastectomies: How to avoid skin necrosis? [in French]. Ann Chir Plast Esthet 2008; 53: 208-225.

22. Berry T, Brooks S, Sydow N: Complication rates of radiation on tissue expander and autologous tissue breast reconstruction. Ann Surg Oncol 2010; 17: 202-210.

23. NCCN Clinical Practice Guidelines in Oncology. Breast Cancer. V.2.2016. Available online: http://www.nccn.org/professionals/physician_ gls/pdf/breast.pdf 